\title{
Simple Sequence Repeat Marker Analysis of Genetic Relationships within Hydrangea macrophylla
}

\author{
Sandra M. Reed ${ }^{1}$ \\ USDA-ARS, Floral and Nursery Plants Research Unit, Tennessee State University Otis L. Floyd \\ Nursery Research Center, 472 Cadillac Lane, McMinnville, TN 37110 \\ Timothy A. Rinehart \\ USDA-ARS, Southern Horticultural Laboratory, 810 Highway 26 West, Poplarville, MS 39470
}

\begin{abstract}
AdDITIONAL INDEX wORDS. SSR, breeding, molecular markers, bigleaf hydrangea, mountain hydrangea, H. serrata
AbStract. Genetic diversity studies using 39 simple-sequence repeat (SSR) markers were carried out with 114 taxa of Hydrangea macrophylla (Thunb.) Ser., including $87 \mathrm{H}$. macrophylla ssp. macrophylla cultivars and 20 members of H. macrophylla ssp. serrata (Thunb.) Makino. The SSR loci were highly variable among the taxa, producing a mean of 8.26 alleles per locus. Overall allelic richness was relatively high at 5.12 alleles per locus. H. macrophylla ssp. serrata contained nearly twice the allelic diversity of $H$. macrophylla ssp. macrophylla. The majority of genetic diversity was found to reside within the subspecies, with only $12 \%$ of the total genetic diversity observed occurring between subspecies. Although the elevation of $\boldsymbol{H}$. macrophylla ssp. serrata to species level has recently been recommended by several hydrangea authorities, these data support the subspecies designation. Four cultivars (Preziosa, Pink Beauty, Tokyo Delight, and Blue Deckle) appeared to be hybrids between the two subspecies. Genetic similarities were found among five remontant cultivars (Bailmer, Oak Hill, David Ramsey, Decatur Blue, and Penny Mac) and several nonremontant cultivars, including General Vicomtesse de Vibraye, Nikko Blue, All Summer Beauty, and La France. No close genetic relationship was found between the remontant cultivar Early Sensation and other remontant cultivars. Genetic similarities were found among variegated and double-flower cultivars. Within H. macrophylla ssp. macrophylla, cultivars with mophead inflorescences clustered separately from most lacecap cultivars. This indicates the cultivars with lacecap inflorescences that were among some of the earliest introductions to Europe were not widely used in the breeding of mophead forms. Some presumed synonyms were found to be valid ('Preziosa' and 'Pink Beauty', 'Rosalba' and 'Benigaku', 'Geoffrey Chadbund' and 'Mowe'), whereas others were not ('Harlequin' and 'Monrey', 'Nigra' and 'Mandschurica'). This study identified potentially unexploited sources of germplasm within $H$. macrophylla and relationships between existing cultivars of this popular shrub. This information should be of value when selecting parents for breeding programs.
\end{abstract}

The genus Hydrangea L. consists of $\approx 23$ species and has an American-Asiatic distribution (McClintock, 1957). Several species are cultivated as ornamentals, the most popular of which is H. macrophylla (Dirr, 2004; van Gelderen and van Gelderen, 2004). This species derives its ornamental appeal from its large corymbs, which consist of a combination of small, inconspicuous perfect flowers and large imperfect flowers with showy sepals. Although the showy flowers are often referred to as sterile, many produce anthers with fully functional pollen (Reed, 2005). In some plants, the inflorescence is globose and only the imperfect flowers are visible; these are referred to as mopheads or hortensias. Lacecaps have flattened inflorescences with many perfect flowers in the center surrounded by a ring of imperfect flowers. Flower color ranges from white to pink to blue, depending on cultivar and availability of aluminum in the soil. Hydrangea macrophylla is cultivated both as a garden plant and a pot plant. In the landscape, it grows 0.9 to $1.8 \mathrm{~m}$ in height with a similar spread,

Received for publication 12 Oct. 2006. Accepted for publication 26 Jan. 2007. David Creech and Barbara Stump at the Mast Arboretum at Stephen F. Austin State University, Kristin VanHoose at Bell Family Nursery, and Michael Dirr at the University of Georgia graciously donated tissue and plants from their collections for this research.

Mention of trade names or commercial products in this article is solely for the purpose of providing specific information and does not imply recommendation or endorsement by the U.S. Department of Agriculture.

${ }^{1}$ Corresponding author. E-mail: Sandra.Reed@ars.usda.gov. and flowers in early to mid summer. As a florist's crop, it is forced into bloom in a greenhouse, usually in time for Easter and Mother's Day sales (Bailey, 1989).

The taxonomic treatment of Hydrangea subsection Macrophyllae E.M. McClint. has long been disputed. Hydrangea macrophylla and $H$. serrata (Thunb.) Ser., the sole members of the subsection, were initially considered to be closely related species (Wilson, 1923), but were combined into a single species by Makino (Hara, 1955). Haworth-Booth (1984) proposed that $H$. macrophylla was a complex hybrid produced from hybridizations of a coastal species with three woodland species. Because the traits used to differentiate the four species were primarily ecological and cultural responses, McClintock (1957) did not support Haworth-Booth's reclassification of the subsection or a hybrid origin for $H$. macrophylla. McClintock (1957) recognized four subspecies of $H$. macrophylla in her taxonomic treatment of the genus. The subspecies commonly known as bigleaf, garden, french, or florist hydrangea, $H$. macrophylla ssp. macrophylla, is native to Japan and is found in coastal areas from sea level to $150 \mathrm{~m}$. Hydrangea macrophylla ssp. serrata, which is cultivated primarily as a garden plant, is found in Japan and northern Korea and is referred to as mountain hydrangea. As the common name indicates, $H$. macrophylla ssp. serrata is usually found in mountainous areas at elevations of 70 to $1500 \mathrm{~m}$. McClintock (1957) differentiated H. macrophylla ssp. macrophylla and $H$. macrophylla ssp. serrata by their leaf and inflorescence sizes, and Bertrand 
(2000) was able to separate the two subspecies using a combination of 28 qualitative and five quantitative morphological criteria. Two other subspecies, H. macrophylla ssp. stylosa (Hook. F. and Thomson) E.M. McClint. and H. macrophylla ssp. chungii (Rehder) E.M. McClint., are native to the eastern Himalayas and southern China and not economically significant (Dirr, 2004; McClintock, 1957; van Gelderen and van Gelderen, 2004).

Many researchers currently believe that $H$. macrophylla ssp. serrata should be elevated to the species level. The use of the epithet $H$. serrata is common in nursery catalogs and recent books dealing with the genus (Dirr, 2004; Mallet, 1994; Mallet et al., 1992; van Gelderen and van Gelderen, 2004). Although morphological differences between $H$. macrophylla ssp. macrophylla and $H$. macrophylla ssp. serrata cultivars are the primary support for the separation of the species, Zonneveld (2004) found that the mean nuclear DNA content of 16 diploid $H$. macrophylla ssp. macrophylla $(2 n=2 x=36)$ cultivars was $5.8 \%$ greater than that of $18 \mathrm{H}$. macrophylla ssp. serrata $(2 n=$ $2 x=36)$ cultivars. This information has been used as support for the species designation for serrata (van Gelderen and van Gelderen, 2004; Zonneveld, 2004). Despite the morphological differences between the subspecies, there are differences in opinion on the taxonomic placement of some cultivars. For example, Dirr (2004) listed 'Miranda' and 'Tokyo Delight' as $H$. serrata cultivars, van Gelderen and van Gelderen (2004) listed them as H. macrophylla, and Bertrand (2001) included them in both the $H$. macrophylla ssp. macrophylla and $H$. macrophylla ssp. serrata cultivar lists. Based on nuclear DNA amount, Zonneveld (2004) proposed that 'Miranda' was a hybrid between $H$. macrophylla and $H$. serrata. A hybrid origin has also been proposed for 'Preziosa', 'Coerulea', 'Acuminata', 'Blue Bird,' f. chinensis, 'Japonica', 'Oamacha', and 'Yae-noAmacha' (Dirr, 2004; Mallet et al., 1992; van Gelderen and van Gelderen, 2004; Zonneveld, 2004).

The history of cultivation of Hydrangea in Asia is not known; however, H. macrophylla was in cultivation in Japan and China long before it was introduced to England and France in the late 19th to early 20th centuries (Haworth-Booth, 1984). Breeding work began in Europe in the early 1900s, resulting in the development of more than 350 cultivars before the beginning of the Second World War. Only limited germplasm was available during that time period to European breeders and, according to Haworth-Booth (1984, p. 146), for 40 years $H$. macrophylla cultivars "have been bred one to another, and in that time almost every possible combination of the available genes has been given birth." Almost all the cultivars developed in Europe during the first half of the 20th century were mophead forms of $H$. macrophylla ssp. macrophylla. Although most were bred for pot plant use, many of these cultivars are used today in the landscape. During its several centuries of cultivation in Japan, numerous cultivars of $H$. macrophylla ssp. serrata (including double-flower, variegated, and colored leaf forms) were selected (Dirr, 2004).

A Hydrangea checklist was published recently that listed $\approx 900 \mathrm{H}$. macrophylla ssp. macrophylla and $160 \mathrm{H}$. macrophylla ssp. serrata cultivars (Bertrand, 2001). About $600 \mathrm{H}$. macrophylla ssp. macrophylla and $140 \mathrm{H}$. macrophylla ssp. serrata extant cultivars have been described (van Gelderen and van Gelderen, 2004), but a recent survey found that only about one-fourth of these cultivars were available in the United States (Dirr, 2004). For some cultivars, nothing has been published about their origins. The breeder, year of release, and awards won are known for many of the cultivars, but pedigree has been identified for only a few (Bertrand, 2001; Haworth-Booth, 1984; van Gelderen and van Gelderen, 2004).

There is confusion within the nursery industry on the identity of many H. macrophylla cultivars, part of which is incited by the use of different names for a single cultivar. Although some synonyms are merely English translations (e.g., 'Blauer Prinz' = 'Blue Prince'), others are not self-explanatory (e.g., 'Mariesii Perfecta' = 'Blue Wave'). To make matters more complicated, some cultivars are offered by nurseries under more than one name. This may occur because there is disagreement among authorities as to which names are synonyms and which represent legitimately different cultivars. Within the United States, there are many plants being grown in landscapes for which the cultivar name or source of the plant is no longer known. Some of these, by virtue of their performance, have drawn the attention of nursery producers who have propagated and released the plants under new cultivar names. At least five remontant cultivars, including the popular cultivar Bailmer (Endless Summer), originated in this manner (Dirr, 2004). Although these remontant cultivars are similar in appearance and appear to be closely related (Lindstrom et al., 2003), their origin is unknown. The physical similarity of many cultivars, along with the variability of flower color of a specific cultivar under different soil conditions, makes correcting H. macrophylla cultivar identification mistakes difficult using phenotype alone.

Microsatellite, or single-sequence repeat (SSR), markers were recently used to study relationships among Hydrangea and related species (Rinehart et al., 2006). Results of that study supported McClintock's classification of the mountain hydrangea as $H$. macrophylla ssp. serrata. Using 14 SSR loci, $21 \mathrm{H}$. macrophylla ssp. macrophylla and $10 \mathrm{H}$. macrophylla ssp. serrata cultivars were found to be $80 \%$ similar. Genetic similarity between $H$. macrophylla ssp. serrata and other species tested was comparable with that between H. macrophylla ssp. macrophylla and those species, which is consistent with a subspecies designation. The objective of the current study, which involves a larger pool of both SSR markers and cultivars, is to evaluate genetic diversity more fully in $H$. macrophylla. We are particularly interested in using the SSR data to study relationships between and within $H$. macrophylla ssp. macrophylla and $H$. macrophylla ssp. serrata, to investigate relationships between and possible origins of remontant cultivars, and to address synonym confusion issues.

\section{Materials and Methods}

Plant materials. Taxa tested in this study are listed in Table 1. We followed McClintock's (1957) taxonomic treatment and used the subspecies designation of $H$. macrophylla ssp. serrata in this paper. Subspecies assignment for cultivars was based on Bertrand (2001), Dirr (2004), Mallet (1994), Mallet et al. (1992), and van Gelderen and van Gelderen (2004). Material tested included $87 \mathrm{H}$. macrophylla ssp. macrophylla and $20 \mathrm{H}$. macrophylla ssp. serrata taxa. Seven cultivars for which the authorities just cited disagreed regarding subspecies assignment or speculated on a hybrid origin were also included. When references differed on spelling of cultivar name, the preferred spelling listed in the cultivar checklist (Bertrand, 2001) was used. Foreign letters in cultivar names have been 
Table 1. List of 114 Hydrangea macrophylla taxa evaluated with 39 single-sequence repeat markers.

\begin{tabular}{|c|c|c|c|c|}
\hline & & Inflorescence & Source of & $\operatorname{Taxa}^{\mathrm{z}}$ \\
\hline $\operatorname{Taxa}^{\mathrm{z}}$ & Subspecies $^{y}$ & type $^{\mathrm{x}}$ & & Little Geisha \\
\hline Akishino Temari & ser & $\mathrm{M}$ & MAST & Maculata \\
\hline All Summer Beauty & mac & M & MAST & Madame Emile Mouillère \\
\hline Altona & mac & M & MAST & Mandshurica \\
\hline Amagi Amacha & ser & $\mathrm{L}$ & MAST & Maréchal Foch \\
\hline Ami Pasquier & $\operatorname{mac}$ & M & Lowe's & Mariesii \\
\hline Ayesha & $\operatorname{mac}$ & M & $\mathrm{BFN}$ & Mariesii Perfecta \\
\hline Bailmer (Endless Summer) & $\operatorname{mac}$ & M & NRC & (Blue Wave) \\
\hline Beauté Vendômoise & $\operatorname{mac}$ & $\mathrm{L}$ & MAST & Mariesii Variegata \\
\hline Benigaku & ser & $\mathrm{L}$ & $\mathrm{BFN}$ & \\
\hline Blauer Prinz (Blue Prince) & mac & M & $\mathrm{NRC}$ & Masja \\
\hline Blaumeise & $\operatorname{mac}$ & $\mathrm{L}$ & NRC & Mathilda Gütges \\
\hline Blue Billow & $\mathrm{mac} / \mathrm{ser}$ & $\mathrm{L}$ & MAST & Merritt's Blue \\
\hline Blue Deckle & ser & $\mathrm{L}$ & MAST & Miranda \\
\hline Bodensee & mac & M & MAST & Miss Belgium \\
\hline f. chinensis & ser & $\mathrm{L}$ & MAST & Miss Hepburn \\
\hline Chiri-san Sue & ser & L, D & MAST & Miyama-yae-Murasaki \\
\hline Coerulea & $\mathrm{mac} / \mathrm{ser}$ & $\mathrm{L}$ & BFN & Monrey (Buttons 'n Bows) \\
\hline David Ramsey & $\operatorname{mac}$ & M & MAST & Mousmee \\
\hline Decatur Blue & mac & M & MAST & Mousseline \\
\hline Dooley & $\operatorname{mac}$ & M & MAST & Möwe \\
\hline $\begin{array}{l}\text { Early Sensation } \\
\quad \text { (Forever and Ever) }\end{array}$ & mac & M & Lowe's & $\begin{array}{l}\text { Nachtigall } \\
\text { Nigra }\end{array}$ \\
\hline Eisvogel & mac & $\mathrm{L}$ & $\mathrm{BFN}$ & Nikko Blue \\
\hline Enziandom & $\operatorname{mac}$ & M & MAST & Oak Hill \\
\hline Fasan & $\operatorname{mac}$ & $\mathrm{L}$ & NRC & Oamacha \\
\hline Forever Pink & $\operatorname{mac}$ & M & $\mathrm{BFN}$ & Oregon Pride \\
\hline Frau Fujiyo (Lady Fujiyo) & $\operatorname{mac}$ & M & UGA & Otaksa \\
\hline Freudenstein & $\operatorname{mac}$ & M & MAST & Parzival \\
\hline Fuji Waterfall (Fujinotaki) & $\mathrm{mac} / \mathrm{ser}$ & L, D & BFN & Penny Mac \\
\hline Gartenbaudirektor Kühnert & $\operatorname{mac}$ & M & Lowe's & Pia \\
\hline $\begin{array}{l}\text { Général Vicomtesse } \\
\text { de Vibraye }\end{array}$ & $\operatorname{mac}$ & M & MAST & $\begin{array}{l}\text { Pink Beauty } \\
\text { Preziosa }\end{array}$ \\
\hline Geoffrey Chadbund & $\operatorname{mac}$ & $\mathrm{L}$ & MAST & Princess Juliana \\
\hline Goliath & $\operatorname{mac}$ & M & MAST & Quadricolor \\
\hline Hamburg & mac & M & MAST & Rosalba \\
\hline Hanabi & mac & L, D & MAST & Rosea \\
\hline Harlequin & $\operatorname{mac}$ & M & MAST & Rotdrössel \\
\hline Heinrich Seidel & $\operatorname{mac}$ & M & NRC & Schenkenburg \\
\hline Hobella & $\operatorname{mac}$ & $\mathrm{L}$ & MAST & Seafoam \\
\hline Horben & $\operatorname{mac}$ & M & NRC & Shamrock \\
\hline Iyo-no-Usuzumi & ser & $\mathrm{L}$ & MAST & Shichidanka \\
\hline Iyo Shibori & ser & $\mathrm{L}$ & MAST & Shishiba \\
\hline Izu-no-Hana & $\operatorname{mac}$ & L, D & MAST & Sir Joseph Banks \\
\hline Jogosaki & mac & L, D & MAST & Skips \\
\hline Kardinal & mac & $\mathrm{L}$ & NRC & Soeur Thérèse \\
\hline Kiyosumi & ser & $\mathrm{L}$ & MAST & Souvenir du Président \\
\hline Kluis Superba & $\operatorname{mac}$ & M & MAST & Doumer \\
\hline Komachi & ser & $\mathrm{M}, \mathrm{D}$ & MAST & Taube \\
\hline Königstein & mac & M & BFN & Thoby \\
\hline La France & $\operatorname{mac}$ & M & NRC & Tiara \\
\hline La Marne & $\operatorname{mac}$ & M & NRC & Tödi \\
\hline Lanarth White & mac & $\mathrm{L}$ & MAST & Tokyo Delight \\
\hline Lemon Wave & $\operatorname{mac}$ & $\mathrm{L}$ & BFN & Tovelit \\
\hline Lemon Zest & $\operatorname{mac}$ & M & MAST & Tricolor \\
\hline Libelle & $\operatorname{mac}$ & $\mathrm{L}$ & MAST & Trophée \\
\hline Lilacina (Mariesii Lilacina) & $\operatorname{mac}$ & $\mathrm{L}$ & MAST & Uzu \\
\hline
\end{tabular}

Table 1. Continued.

\begin{tabular}{|c|c|c|c|}
\hline $\operatorname{Taxa}^{\mathrm{z}}$ & Subspecies ${ }^{\mathrm{y}}$ & $\begin{array}{c}\text { Inflorescence } \\
\text { type }^{\mathrm{x}}\end{array}$ & $\begin{array}{l}\text { Source of } \\
\text { leaf tissue }\end{array}$ \\
\hline Little Geisha & ser & $\mathrm{M}$ & $\mathrm{NRC}$ \\
\hline Maculata & mac & $\mathrm{L}$ & BFN \\
\hline Madame Emile Mouillère & $\mathrm{mac}$ & M & MAST \\
\hline Mandshurica & $\operatorname{mac}$ & M & MAST \\
\hline Maréchal Foch & $\operatorname{mac}$ & M & $\mathrm{NRC}$ \\
\hline Mariesii & mac & $\mathrm{L}$ & VG \\
\hline $\begin{array}{c}\text { Mariesii Perfecta } \\
\text { (Blue Wave) }\end{array}$ & mac & $\mathrm{L}$ & BFN \\
\hline Mariesii Variegata & $\operatorname{mac}$ & $\mathrm{L}$ & $\begin{array}{l}\text { Home } \\
\text { Depot }\end{array}$ \\
\hline Masja & $\operatorname{mac}$ & M & MAST \\
\hline Mathilda Gütges & $\mathrm{mac}$ & M & NRC \\
\hline Merritt's Blue & $\operatorname{mac}$ & M & MAST \\
\hline Miranda & $\mathrm{mac} / \mathrm{ser}$ & $\mathrm{L}$ & MAST \\
\hline Miss Belgium & $\operatorname{mac}$ & M & MAST \\
\hline Miss Hepburn & $\operatorname{mac}$ & M & $\mathrm{BFN}$ \\
\hline Miyama-yae-Murasaki & ser & L, D & MAST \\
\hline Monrey (Buttons 'n Bows) & $\operatorname{mac}$ & M & MAST \\
\hline Mousmee & $\operatorname{mac}$ & $\mathrm{L}$ & MAST \\
\hline Mousseline & $\operatorname{mac}$ & M & MAST \\
\hline Möwe & $\mathrm{mac}$ & $\mathrm{L}$ & MAST \\
\hline Nachtigall & $\operatorname{mac}$ & $\mathrm{L}$ & MAST \\
\hline Nigra & $\operatorname{mac}$ & M & NRC \\
\hline Nikko Blue & mac & M & Lowe's \\
\hline Oak Hill & mac & M & MAST \\
\hline Oamacha & ser & $\mathrm{L}$ & $\mathrm{BFN}$ \\
\hline Oregon Pride & $\operatorname{mac}$ & M & BFN \\
\hline Otaksa & $\mathrm{mac}$ & M & BFN \\
\hline Parzival & $\operatorname{mac}$ & M & MAST \\
\hline Penny Mac & $\mathrm{mac}$ & M & $\mathrm{BFN}$ \\
\hline Pia & $\operatorname{mac}$ & M & BFN \\
\hline Pink Beauty & $\mathrm{mac} / \mathrm{ser}$ & M & MAST \\
\hline Preziosa & $\mathrm{mac} / \mathrm{ser}$ & M & BFN \\
\hline Princess Juliana & $\operatorname{mac}$ & M & BFN \\
\hline Quadricolor & mac & $\mathrm{L}$ & MAST \\
\hline Rosalba & ser & $\mathrm{L}$ & MAST \\
\hline Rosea & $\operatorname{mac}$ & M & VG \\
\hline Rotdrössel & $\mathrm{mac}$ & $\mathrm{L}$ & BFN \\
\hline Schenkenburg & $\operatorname{mac}$ & M & BFN \\
\hline Seafoam & $\operatorname{mac}$ & $\mathrm{L}$ & BFN \\
\hline Shamrock & $\operatorname{mac}$ & L, D & UGA \\
\hline Shichidanka & ser & L, D & NRC \\
\hline Shishiba & ser & $\mathrm{L}$ & MAST \\
\hline Sir Joseph Banks & $\operatorname{mac}$ & M & VG \\
\hline Skips & $\mathrm{mac}$ & M & MAST \\
\hline Soeur Thérèse & $\mathrm{mac}$ & M & MAST \\
\hline $\begin{array}{l}\text { Souvenir du Président } \\
\text { Doumer }\end{array}$ & $\operatorname{mac}$ & M & MAST \\
\hline Taube & $\operatorname{mac}$ & $\mathrm{L}$ & MAST \\
\hline Thoby & ser & $\mathrm{L}$ & MAST \\
\hline Tiara & ser & $\mathrm{L}$ & MAST \\
\hline Tödi & mac & M & $\mathrm{BFN}$ \\
\hline Tokyo Delight & $\mathrm{mac} / \mathrm{ser}$ & $\mathrm{L}$ & MAST \\
\hline Tovelit & mac & M & Lowe's \\
\hline Tricolor & $\operatorname{mac}$ & $\mathrm{L}$ & $\mathrm{BFN}$ \\
\hline Trophée & $\mathrm{mac}$ & M & NRC \\
\hline Uzu & $\mathrm{mac}$ & M & MAST \\
\hline
\end{tabular}


Table 1. Continued.

\begin{tabular}{lccc}
\hline \multicolumn{1}{c}{ Taxa $^{\mathrm{z}}$} & Subspecies $^{\mathrm{y}}$ & $\begin{array}{c}\text { Inflorescence } \\
\text { type }^{\mathrm{x}}\end{array}$ & $\begin{array}{c}\text { Source of } \\
\text { leaf tissue }\end{array}$ \\
\hline Veitchii & mac & $\mathrm{L}$ & NRC \\
White Swan & $\mathrm{mac}$ & $\mathrm{M}$ & MAST \\
$\begin{array}{l}\text { White Wave } \\
\quad \text { Mariesii Grandiflora) }\end{array}$ & $\mathrm{mac}$ & $\mathrm{L}$ & UGA \\
Woodlander & ser & $\mathrm{L}$ & MAST \\
Yae-no-Amacha & ser & L, D & MAST \\
Zaunköenig & $\mathrm{mac}$ & $\mathrm{L}$ & NRC \\
\hline
\end{tabular}

${ }^{\mathrm{z}}$ Names in parentheses indicate synonyms or trademarked names. Synonyms are provided only when they are English translations or confirmed renaming of cultivars; the synonym listed first is the name under which we acquired the plant.

${ }^{\mathrm{y}} \mathrm{mac}, H$. macrophylla ssp. macrophylla; ser, H. macrophylla ssp. serrata; mac/ser, sources differ on subspecies assignment or cultivar has been proposed to be a hybrid between the two subspecies.

${ }^{x} \mathrm{D}$, imperfect flowers have multiple layers of sepals, giving doubleflower appearance; L, lacecap; M, mophead.

${ }^{w}$ BFN, Bell Family Nursery, Aurora, Ore.; MAST, Stephen F. Austin MAST Arboretum, Nacogdoches, Texas; NRC, Tennessee State University Nursery Research Center, McMinnville, Tenn.; UGA, University of Georgia, Athens, Ga.; VG, Vintage Gardens, Sebastopol, Calif.

Subspecies assignment, inflorescence type, and source of tissue are included.

eliminated in text and figures, but are included in Table 1. Synonyms are provided in Table 1 only when they are English translations of a foreign name or confirmed renaming of cultivars (Haworth-Booth, 1984). When valid synonyms exist, we refer to the plant under the name by which we acquired it. Source of tissue and inflorescence form of each taxa are also presented in Table 1. Three taxa of $H$. scandens (L. f.) Ser. were included in the analysis for rooting the phenogram.

A single representative plant was used for $104 \mathrm{H}$. macrophylla taxa. Two or three plants each of 'Ami Pasquier', 'Benigaku', 'Blaumeise', 'Bailmer', 'Nikko Blue', 'Oregon Pride', 'Pink Beauty', 'Preziosa', 'Taube', and 'Veitchii' were analyzed. Because all duplicate samples yielded comparable data, data from only one representative of those cultivars is presented.

SSR DEVELOPMENT AND SAMPLe PROCessing. Previously described SSR-enriched libraries (Rinehart et al., 2006) were screened for polymorphic loci against a panel of eight cultivars. DNA was extracted from $1 \times 1-\mathrm{cm}$ pieces of fresh leaf tissue using the Qiagen Plant Mini Kit (Qiagen, Valencia, Calif.) and was quantified using a NanoDrop Spectrophotometer (NanoDrop Technologies, Wilmington, Del.). Singlesequence repeat amplification was performed using a modified three-primer protocol (Rinehart et al., 2006). Fluorescencelabeled polymerase chain reaction (PCR) fragments were visualized by automated capillary gel electrophoresis on an ABI3100-Avant or ABI3730xl using ROX-500 size standard (Applied Biosystems, Foster City, Calif.). GeneMapper version 3.7 was used to recognize and size peaks (Applied Biosystems). We will release DNA sequence data including primer sequences after placement on a genome map for H. macrophylla.

Data ANALYSIS. Data from 39 SSR loci were compiled for the 114 taxa and analyzed for shared allele frequencies. Nei's (1972) minimum genetic distance and standard genetic distance (Ds) were calculated for all samples. Gene diversity estimates were produced using Nei's 1987 estimator for heterozygosity and unbiased gene diversity per population was determined using FSTAT software v.1.2 (Goudet, 1995; Saitou and Nei, 1987). Allele sharing statistics were used independent of ploidy differences, and all alleles were represented as diploid. Among-population variation was calculated for each subspecies by comparing effective numbers of alleles with differences in allele frequencies between taxa. Average gene diversity was calculated between populations (Dst) and calculated relative to total gene diversity (Gst) (Nei, 1973).

POPULATIONS version 1.2.28 was used for phenetic analyses (Langella, 2002). Genetic distances between individual samples were calculated using allele sharing distance to create a distance matrix (Jin and Chakraborty, 1994; Stephens et al., 1992). Principal coordinate analysis (PCoA) plots and tree dendograms were based on the allele sharing distance matrix, which included missing data as null alleles. Principal coordinate analysis was performed using NTSys software (Rohlf, 1992). Neighbor joining with 100 bootstrap replicates for statistical support was used to generate a tree phenogram showing clustering of genetically similar samples (Saitou and Nei, 1987). The phenogram was visualized with TreeView (Page, 1996).

\section{Results}

The 39 SSR loci were highly variable among the H. macrophylla taxa. Number of alleles per locus ranged from three (STAB061_062 and STAB363_364) to 26 (STAB227_228), with a mean of 8.26 alleles per locus (Table 2). All but one of the repeats [STAB647_648; TTTA(7)] were trinucleotide, and 31 produced a range of actual allele sizes that included the predicted size. Of the 4446 PCR amplifications attempted, only 76 failed to produce usable data despite repeated attempts. Failures were three times more likely within $H$. macrophylla ssp. serrata, for which each sample averaged 1.5 failures (3.8\%), than in $H$. macrophylla ssp. macrophylla, which averaged 0.42 failures (1.1\%). Data were missing from at least one locus for 38 of the taxa, but no single cultivar accounted for the majority of missing data in either subspecies. Cultivars missing data for more than two loci (followed by number of primers failing to generate data) were Ami Pasquier (7), General Vicomtesse de Vibraye (4), Chiri-san Sue (4), Thoby (4), Iyo Shibori (3), Miranda (3), and, Kiyosumi (3). Likewise, missing data could not be attributed to poor amplification of a single locus as four loci-STAB107_108, STAB409_410, STAB347_348, and STAB317_318_failed most often with $16,14,8$, and 4 failures respectively.

The PCoA based on gene frequencies of all samples is presented in Fig. 1. The PCoA accounted for $38.7 \%$ of the total variation. Two main clusters were discernible, one containing H. macrophylla ssp. serrata and the other H. macrophylla ssp. macrophylla cultivars. Similar results are seen in the phenogram derived from allele sharing frequencies (Fig. 2). Division between $H$. macrophylla ssp. serrata and H. macrophylla ssp. macrophylla cultivars is clearly visible, but bootstrap support was low (33\%) for the basal node that divides the tree into subspecies (data not shown). Seven of the cultivars in this study were listed in Table 1 as possible hybrids or of disputed subspecies assignment. Three of these cultivars, Blue Billow, Coerulea, and Miranda, fell into the H. macrophylla ssp. 
Table 2. Characteristics of 39 single-sequence repeat (SSR) loci used in the analysis of 114 Hydrangea macrophylla taxa.

\begin{tabular}{|c|c|c|c|c|c|c|c|c|c|c|c|c|}
\hline \multirow[b]{3}{*}{ Locus $^{\mathrm{z}}$} & \multirow{3}{*}{$\begin{array}{c}\text { Repeat } \\
\text { motif }\end{array}$} & \multirow{3}{*}{$\begin{array}{l}\text { Expected } \\
\text { allele } \\
\text { size (bp) }\end{array}$} & \multirow{3}{*}{$\begin{array}{c}\text { Actual } \\
\text { allele size } \\
\text { range (bp) }\end{array}$} & \multirow{3}{*}{$\begin{array}{l}\text { Alleles } \\
\text { (n) }\end{array}$} & \multicolumn{3}{|c|}{ Allelic richness ${ }^{\mathrm{y}}$} & \multirow{2}{*}{\multicolumn{5}{|c|}{ Gene diversity $^{\mathrm{x}}$}} \\
\hline & & & & & ubspecies & Subspecies & & & & & & \\
\hline & & & & & macrophylla & serrata & All & Ho & $\mathrm{Ht}$ & Hs & Dst & $\overline{\text { Gst }}$ \\
\hline$\overline{\text { STAB045_046 }^{\dagger}}$ & TCA(8) & 15 & $135-150$ & 6 & 2.998 & 5.840 & 4.711 & 0.585 & 0.758 & 0.683 & 0.075 & $\overline{0.099}$ \\
\hline STAB061_062 ${ }^{\dagger}$ & $\mathrm{CAC}(4)$ & 96 & $86-97$ & 3 & 1.948 & 2.900 & 2.351 & 0.244 & 0.257 & 0.257 & 0.001 & 0.002 \\
\hline STAB071_072 & TGA(8) & 140 & $126-148$ & 7 & 1.995 & 5.364 & 3.452 & 0.392 & 0.412 & 0.400 & 0.012 & 0.028 \\
\hline STAB091_092 & $\operatorname{AGA}(12)$ & 160 & $138-173$ & 14 & 6.130 & 5.965 & 7.852 & 0.732 & 0.726 & 0.696 & 0.029 & 0.041 \\
\hline STAB107_108 & CAT(8) & 142 & $128-149$ & 6 & 1.982 & 5.782 & 4.485 & 0.294 & 0.699 & 0.466 & 0.233 & 0.334 \\
\hline STAB113_114 & $\mathrm{TCA}(8)$ & 122 & $108-121$ & 5 & 2.697 & 3.612 & 3.254 & 0.274 & 0.363 & 0.354 & 0.009 & 0.024 \\
\hline STAB125_126 ${ }^{\dagger}$ & CTT(4) & 136 & $145-160$ & 6 & 1.927 & 5.958 & 4.677 & 0.426 & 0.673 & 0.506 & 0.168 & 0.249 \\
\hline STAB137_137 & ATC(10) & 148 & $102-146$ & 11 & 4.392 & 8.476 & 6.898 & 0.602 & 0.843 & 0.771 & 0.072 & 0.085 \\
\hline STAB161_162 & $\mathrm{CAG}(7)$ & 82 & $65-88$ & 5 & 2.842 & 4.339 & 3.393 & 0.520 & 0.597 & 0.572 & 0.025 & 0.042 \\
\hline STAB165_166 & $\mathrm{CAC}(6)$ & 149 & $145-155$ & 5 & 2.860 & 4.077 & 3.279 & 0.438 & 0.668 & 0.515 & 0.154 & 0.230 \\
\hline STAB173_174 & $\mathrm{TCA}(8)$ & 82 & 79-97 & 5 & 2.917 & 3.394 & 3.331 & 0.538 & 0.550 & 0.548 & 0.002 & 0.003 \\
\hline STAB181_182 & TTC(12) & 158 & $130-170$ & 21 & 7.098 & 12.544 & 10.970 & 0.902 & 0.911 & 0.869 & 0.042 & 0.046 \\
\hline STAB193_194 & TTC(12) & 149 & $120-149$ & 6 & 3.064 & 3.646 & 3.501 & 0.424 & 0.364 & 0.350 & 0.015 & 0.041 \\
\hline STAB227_228 & TTC(12) & 155 & $81-175$ & 26 & 5.108 & 15.428 & 9.997 & 0.822 & 0.873 & 0.794 & 0.080 & 0.091 \\
\hline STAB239_240 & $\operatorname{AAG}(8)$ & 159 & $141-173$ & 11 & 4.759 & 9.556 & 7.196 & 0.727 & 0.861 & 0.824 & 0.037 & 0.043 \\
\hline STAB241_242 & TTC(10) & 120 & $94-129$ & 10 & 5.110 & 8.291 & 7.378 & 0.745 & 0.828 & 0.751 & 0.077 & 0.093 \\
\hline STAB247_248 & $\operatorname{AAG}(5)$ & 127 & $117-145$ & 8 & 2.968 & 6.318 & 5.077 & 0.477 & 0.747 & 0.640 & 0.107 & 0.143 \\
\hline STAB259_260 & $\operatorname{AGA}(5)$ & 131 & $100-135$ & 10 & 4.864 & 6.512 & 6.268 & 0.659 & 0.748 & 0.701 & 0.047 & 0.063 \\
\hline STAB271_272 & CAG(7) & 142 & $126-141$ & 5 & 2.447 & 4.108 & 3.057 & 0.477 & 0.509 & 0.444 & 0.065 & 0.128 \\
\hline STAB305_306 & CAG(8) & 143 & $123-144$ & 8 & 3.992 & 6.459 & 5.713 & 0.818 & 0.804 & 0.717 & 0.087 & 0.108 \\
\hline STAB313_314 & TGC(10) & 156 & $141-163$ & 7 & 3.559 & 4.991 & 4.597 & 0.377 & 0.695 & 0.529 & 0.166 & 0.238 \\
\hline STAB317_318 & AAG(8) & 150 & $141-151$ & 8 & 2.890 & 5.987 & 4.527 & 0.458 & 0.721 & 0.602 & 0.119 & 0.165 \\
\hline STAB321_322 ${ }^{\dagger}$ & $\mathrm{TCT}(7)$ & 159 & $145-164$ & 7 & 3.650 & 5.546 & 4.909 & 0.635 & 0.763 & 0.679 & 0.084 & 0.110 \\
\hline STAB347_348 & TCA(8) & 154 & $142-169$ & 10 & 4.710 & 7.085 & 6.413 & 0.737 & 0.758 & 0.705 & 0.053 & 0.070 \\
\hline STAB351_352 & $\operatorname{ATG}(8)$ & 159 & $149-176$ & 13 & 2.851 & 10.881 & 6.615 & 0.314 & 0.734 & 0.603 & 0.131 & 0.179 \\
\hline STAB363_364 & ATC(8) & 99 & $82-94$ & 3 & 2.619 & 1.600 & 2.643 & 0.324 & 0.460 & 0.272 & 0.188 & 0.409 \\
\hline STAB379_380 & ATC(6) & 113 & $102-114$ & 4 & 1.998 & 3.424 & 2.510 & 0.355 & 0.542 & 0.334 & 0.208 & 0.384 \\
\hline STAB389_390 & GAT(8) & 88 & $70-92$ & 8 & 4.119 & 5.538 & 5.620 & 0.601 & 0.755 & 0.680 & 0.074 & 0.098 \\
\hline STAB409_410 & ATG(8) & 156 & $144-169$ & 5 & 2.948 & 5.000 & 3.421 & 0.321 & 0.414 & 0.412 & 0.002 & 0.004 \\
\hline STAB413_414 & CAG(6) & 88 & $62-98$ & 7 & 3.232 & 6.535 & 4.854 & 0.665 & 0.737 & 0.704 & 0.033 & 0.044 \\
\hline STAB421_422 & CAG(8) & 150 & $131-149$ & 7 & 4.497 & 5.417 & 5.599 & 0.687 & 0.799 & 0.723 & 0.077 & 0.096 \\
\hline STAB423_424 & GCT(8) & 140 & $114-155$ & 10 & 4.647 & 7.340 & 6.304 & 0.588 & 0.782 & 0.707 & 0.075 & 0.096 \\
\hline STAB429_430 ${ }^{\dagger}$ & $(\mathrm{CTG}) 6$ & 83 & $69-84$ & 6 & 2.000 & 5.002 & 3.355 & 0.506 & 0.631 & 0.538 & 0.093 & 0.148 \\
\hline STAB445_446 & $\operatorname{AAG}(8)$ & 140 & $132-159$ & 12 & 3.893 & 8.022 & 5.996 & 0.528 & 0.723 & 0.653 & 0.070 & 0.097 \\
\hline STAB501_502 ${ }^{\dagger}$ & $\mathrm{CAA}(4)$ & 118 & $93-111$ & 5 & 1.999 & 3.938 & 2.552 & 0.269 & 0.543 & 0.362 & 0.181 & 0.334 \\
\hline STAB539_540 & CTG(6) & 138 & $125-169$ & 11 & 5.197 & 8.588 & 8.006 & 0.644 & 0.865 & 0.769 & 0.096 & 0.111 \\
\hline STAB567_568 & GAT(8) & 158 & $142-164$ & 8 & 3.252 & 6.256 & 5.355 & 0.629 & 0.797 & 0.720 & 0.077 & 0.097 \\
\hline STAB619_620 & TGA(4) & 139 & $134-146$ & 4 & 2.135 & 3.143 & 3.111 & 0.265 & 0.443 & 0.418 & 0.025 & 0.057 \\
\hline STAB647_648 & TTTA(7) & 134 & $101-139$ & 14 & 4.848 & 7.584 & 6.318 & 0.636 & 0.717 & 0.628 & 0.089 & 0.124 \\
\hline
\end{tabular}

${ }^{\mathrm{z} S S R}$ loci previously described (Rinehart et al., 2006) indicated by a single dagger.

${ }^{\mathrm{y}}$ Allelic richness calculated for all samples and for $H$. macrophylla ssp. macrophylla and $H$. macrophylla ssp. serrata separately.

${ }^{x}$ Dst, gene diversity between $H$. macrophylla ssp. macrophylla and $H$. macrophylla ssp. serrata; Gst, proportion of gene diversity maintained among subspecies, or proportion of total diversity that is the result of differences in allele frequencies among populations (also called the coefficient of differentiation, ranging from 0 to 1 where 0 indicates all variation maintained within the subspecies and 1 indicates all variation is among subspecies); Ho, observed proportion of heterozygotes for all samples; Hs, gene diversity within H. macrophylla ssp. macrophylla and H. macrophylla ssp. serrata; Ht, total gene diversity.

serrata cluster, whereas 'Fuji Waterfall' was in the H. macrophylla ssp. macrophylla cluster. The remaining three cultivars of uncertain taxonomic status-Pink Beauty, Preziosa, and Tokyo Delight-along with the H. macrophylla ssp. serrata cultivar Blue Deckle, were situated between the two main clusters but closer to H. macrophylla ssp. macrophylla than to $H$. macrophylla ssp. serrata.

Allelic richness was calculated overall and for both subspecies (Table 2). Overall richness was relatively high at 5.12 alleles per locus. Hydrangea macrophylla ssp. serrata con- tained nearly twice the allelic diversity of $H$. macrophylla ssp. macrophylla, with 6.16 and 3.52 alleles per locus respectively. Observed heterozygosity varied from 0.244 to 0.902 , with an average of 0.529 across all loci, which suggests a fairly even split between homozygous and heterozygous loci (Table 2). Genetic structure between the subspecies was assessed by partitioning the total gene diversity $(\mathrm{Ht}=0.668)$ into within subspecies $(\mathrm{Hs}=0.587)$ and between subspecies $(\mathrm{Dst}=0.081)$ diversity. The majority of gene diversity resides within the subspecies, as supported by an average Gst value of 0.122 . This 


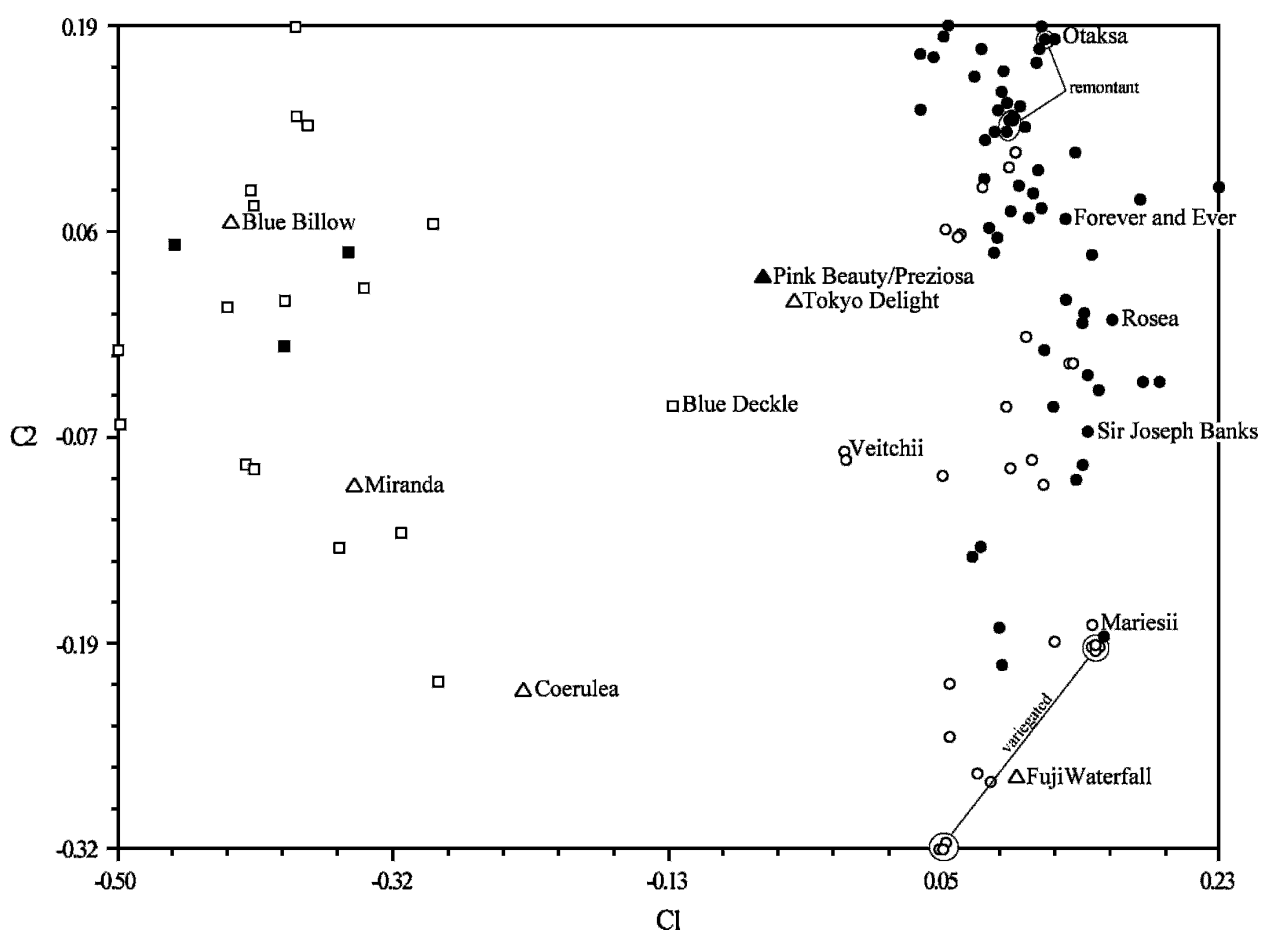

Fig. 1. Principal coordinate analysis of 114 Hydrangea macrophylla taxa derived from allele sharing distance matrix. Circles indicate $H$. macrophylla ssp. macrophylla. Squares represent $H$. macrophylla ssp. serrata. Taxa for which subspecies assignment is disputed, as indicated in Table 1, are shown as triangles. Mophead inflorescences are indicated by darkened/filled circles, squares, or triangles whereas cultivars with lacecap inflorescences are unshaded. Variegated and remontant cultivars are circled.

suggests that only about $12 \%$ of the total genetic diversity that we observed occurs between subspecies.

Average pairwise divergence for the entire group of 114 taxa was $32.7 \%$ using Nei's minimum genetic distance (Nei, 1972). Of all taxa sampled, 'Iyo Shibori' and 'Soeur Therese' exhibited the most divergence $(70.1 \%)$. For analyses within and between subspecies, we removed 'Blue Deckle', 'Pink Beauty', 'Preziosa', and 'Tokyo Delight', because they are potential hybrids, and grouped samples as $H$. macrophylla ssp. macrophylla or $H$. macrophylla ssp. serrata based on their position in Fig. 1. Average pairwise divergence within $H$. macrophylla ssp. macrophylla was 24\%, with 'Miss Belgium' and 'Shamrock' showing the greatest genetic divergence (52.6\%). Average pairwise divergence within H. macrophylla ssp. serrata was $41 \%$, with the greatest divergence between 'Komachi' and 'Kiyosumi' (56.3\%). Minimum genetic distance between subspecies was $17.2 \%$.

Identical genotypes were found at all 39 loci examined in the following four sets of cultivars: Blaumeise and Eisvogel; Lemon Wave, Maculata, Mariesii Perfecta, and Seafoam; Fasan and Zaunkoenig; and Pink Beauty and Preziosa. Identical genotypes were also found in five other sets of cultivars but, because of missing data for one or more loci, bootstrap support for the relationships was less than $100 \%$. These groups of cultivars were David Ramsey, Bailmer, and Oak Hill; Geoffrey Chadbund, Kardinal, and Mowe; Mariesii Variegata and Tricolor; General Vicomtesse de Vibraye and Kluis Superba; and Nikko Blue, Penny Mac, and Otaska.

The remontant cultivars David Ramsey, Oak Hill, Bailmer, and Decatur Blue grouped together and were related to a clade containing the cultivars General Vicomtesse de Vibraye, Kluis
Superba, and La France (Figs. 1 and 2). These two clades were related to a third clade consisting of the remontant cultivar Penny Mac along with Otaska, Nikko Blue, All Summer Beauty, and Dooley. Another remontant cultivar, Early Sensation (Forever and Ever), did not cluster with the other remontant cultivars, but was in a clade with cultivars Bodensee and Merritt's Blue, both of which are popular cultivars for greenhouse forcing.

The variegated $H$. macrophylla ssp. macrophylla cultivars were represented in two clades (Figs. 1 and 2 ). One clade contained the variegated cultivars Lemon Wave and Maculata along with the nonvariegated cultivars Mariesii Perfecta, Seafoam, Mousseline, and Mariesii. The other variegated clade contained only the variegated cultivars Mariesii Variegata, Tricolor, and Quadricolor.

In addition to the remontant and variegated clades, several other sets of cultivars appeared genetically similar with good bootstrap support for the relationships (Fig. 2). Nine members of the Teller Series of lacecap cultivars fell into three clades. One consisted of 'Fasan' and 'Zaunkoenig'; another of 'Nachtigall', 'Taube', 'Blaumeise', and 'Eisvogel'; and the third of 'Geoffrey Chadbund', 'Mowe', and 'Kardinal'. The other two Teller Series cultivars that were included in this study, Rotsdrossel and Libelle, did not exhibit close genetic similarity to any other cultivars in this series. 'Ayesha' and 'Uzu' were almost 100\% identical and were similar to 'Sir Joseph Banks'. Close genetic similarity was also observed in the following sets of $H$. macrophylla ssp. macrophylla cultivars: Freudenstein and Masja, Ami Pasquier and Souvenir du President Doumer, Altona and Goliath, Mandschurica and Nigra, and Hanabi and Izo-no-Hana. Two H. macrophylla ssp. serrata cultivars, Rosalba and Benigaku, also clustered closely.

Within the $H$. macrophylla ssp. macrophylla cluster, most mophead cultivars clustered separately from the majority of lacecap cultivars (Fig. 1). The exception to this was 10 Teller Series lacecap cultivars that were within the mophead cluster, and five mophead cultivars that were outside the main mophead cluster. Three ('Madame Emile Mouillere', 'Princess Juliana', and 'Soeur Therese') of the four white-flower mophead cultivars in this study were in this outlying mophead group. 'Rosea', 'Otaska', and 'Sir Joseph Banks', which were the original mophead cultivars imported to Europe from Asia in the late 19th to early 20th century (Haworth-Booth, 1984), were situated within the large $H$. macrophylla ssp. macrophylla mophead cluster. In contrast, the first lacecap cultivars to be imported to Europe, Veitchii and Mariessi, were some distance from the mophead cluster.

With the exception of 'Fasan', 'Libelle', and 'Zaunkoenig', all the Teller series cultivars examined in this study are triploids 


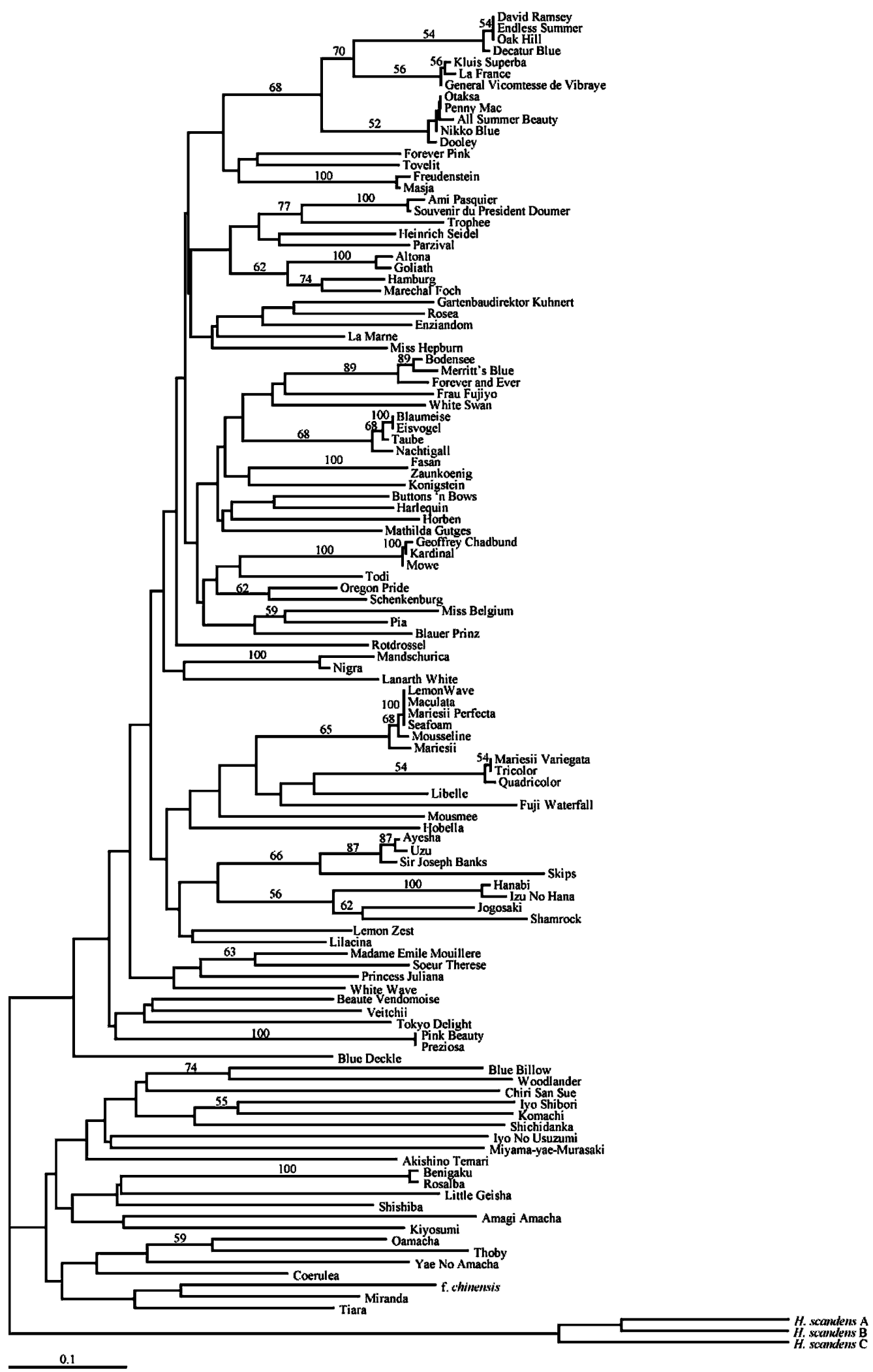

Fig. 2. Neighbor-joining phenogram generated from allele sharing distance matrix analysis based on the proportion of shared alleles at 39 single-sequence repeat loci among 114 Hydrangea macrophylla taxa. Bootstrap values out of 100 replicates are shown if 50\% or higher. Tree was rooted with (A) H. scandens ssp. chinensis (Maxim.) E.M. McClint. f. angustipetala (syn. H. angustipetala Hayata), (B) H. scandens ssp. chinensis f. lobbii (syn. H. lobbii Maxim.), and (C) H. scandens ssp. liukiuensis (Nakai) E.M. McClint. (syn. H. luteovenosa Koidzumi). 
(Zonneveld, 2004; Jones et al., in press). Other known triploids included in this study were 'Enziandom', 'Miss Hepburn', 'Heinrich Seidel', 'Altona', 'Goliath', 'Hamburg', 'Marechal Foch', 'Oregon Pride', 'Schenkenburg', and 'Masja'. The relationships between 'Altona' and 'Goliath', and among the Teller Series cultivars have already been mentioned. Among the other triploids, 'Hamburg' and 'Marechal Foch' showed genetic similarity, as did 'Oregon Pride' and 'Schenkenburg'.

\section{Discussion}

Results of this study support the subspecies designation for the mountain hydrangea as genetic diversity estimates indicated that there was about eight times more diversity within the subspecies than there was between them. Differences in genome size between $H$. macrophylla ssp. macrophylla and $H$. macrophylla ssp. serrata have been used to support splitting them into separate species (van Gelderen and van Gelderen, 2004; Zonneveld, 2004). However, because intraspecific differences in genome size among populations of plant species collected from different geographical areas are not uncommon (Levin, 2002), we feel that the SSR data present a strong case for keeping serrata at the subspecies level.

There does not appear to have been much interbreeding between $H$. macrophylla ssp. macrophylla and H. macrophylla ssp. serrata. In nature the two subspecies are geographically isolated, so possibilities of intersubspecies mating would have been limited to garden settings or to controlled pollinations. Four possible hybrids were identified, but no well-documented information regarding the origins of any of these is available. Pollen staining indicated a high level of fertility in two of these cultivars: Tokyo Delight and Preziosa (Reed and Rinehart, 2006). Although we have only worked with a limited number of parental cultivars, we have successfully produced fertile hybrids between the subspecies (Reed, 2000; S.M. Reed, unpublished data). Assuming hybridizations are possible using a wide range of cultivars of both subspecies, intersubspecies hybridization appears to be an unexploited route toward improvement of $H$. macrophylla.

As we found in an earlier study (Rinehart et al., 2006), there is more genetic diversity within $H$. macrophylla ssp. serrata than within $H$. macrophylla ssp. macrophylla. This is no doubt because most of the $H$. macrophylla ssp. serrata cultivars were selected from the wild, whereas most of the H. macrophylla ssp. macrophylla cultivars were developed through breeding efforts using a small number of original, imported cultivars. With the exception of 'Benigaku' and 'Rosalba', which will be discussed later, none of the H. macrophylla ssp. serrata cultivars examined showed high levels of genetic similarity. Even cultivars such as Akishino Temari, Iyo-no-Usuzumi, and Iyo Shibori, which were collected from the same general area (Yamamoto, 2000), show considerable genetic divergence. The full scope of the diversity of this subspecies is most likely much greater than was revealed in this study because there is considerable wild and cultivated H. macrophylla ssp. serrata germplasm in Japan and Korea that has not been imported to Europe or the United States.

In contrast to $H$. macrophylla ssp. serrata, a high level of genetic similarity was found within groups of $H$. macrophylla ssp. macrophylla cultivars. This study involved six cultivars that are reported to flower on the current year's growth, which allows them to flower even if a cold winter or spring freeze destroys above-ground growth. Because they also have the potential to flower more than once during the summer, these cultivars have been termed remontant. Five remontant cultivars (Bailmer, Decatur Blue, David Ramsey, Penny Mac, and Oak Hill) are similar in appearance and, at one time, it was thought that they all represented the same pot plant cultivar that had been planted in gardens in different locations (Dirr, 2004). Analysis with randomly amplified polymorphic DNA markers showed no differences between 'Bailmer' and 'David Ramsey', both of which produced banding patterns very similar to those of 'Decatur Blue' and 'Oak Hill', whereas 'Penny Mac' was in a separate clade along with 'Dooley' and 'Nikko Blue' (Lindstrom et al., 2003). Our results were in general agreement with that study, although we found no differences between 'Bailmer', 'David Ramsey', and 'Oak Hill'. However, data from additional loci suggest that SSRs can discriminate between all the remontant cultivars, including Bailmer, David Ramsey, and Oak Hill (data not presented). Therefore, it appears that all remontant cultivars tested in this study are distinct cultivars, but that the cultivars Bailmer, Oak Hill, David Ramsey, and Decatur Blue are very similar genetically. There is also considerable genetic similarity between these four cultivars and another remontant cultivar, Penny Mac.

'Nikko Blue', 'All Summer Beauty', 'Dooley', 'General Vicomtesse de Vibraye', 'Otaksa', 'La France', and 'Kluis Superba' were found to share considerable genetic similarity with the five aforementioned remonant cultivars. With the exception of 'Kluis Superba', all these cultivars are described as free flowering (Dirr, 2004; Haworth-Booth, 1984), indicating that they produce flowers not only from terminal shoots, but also from lateral shoots arising along the full length of the stem. Cultivars that produce flowers from basal shoots may flower after freezing temperatures kill new shoots arising from upper parts of the stems, but they do not have the ability to flower repeatedly throughout the summer. 'Kluis Superba' also differs from the other cultivars in this clade because it produces deeply colored flowers. The inclusion of 'Otaska' in the remontant clade is of particular interest because it was one of the first cultivars introduced from Japan to Europe, and was used extensively in the breeding of the European cultivars (Haworth-Booth, 1984). It would be prudent to evaluate additional 'Otaska' specimens from different sources to confirm this relationship.

'Early Sensation', which was introduced to the United States in 2005 and is being marketed as being remontant, was not genetically similar to the other remontant cultivars. However, Dirr (2004) states that 'Bodensee', which we found to be genetically similar to 'Early Sensation', typically blooms twice in one season. It is not known whether 'Early Sensation' represents a source of different genes for remontancy or the same genes in a different genetic background. In either instance, it should be considered as a parent in breeding programs.

We also observed genetic similarities among variegated cultivars. All five variegated cultivars that were included in this study produce lacecap inflorescences and are of uncertain origin. 'Mariesii Variegata' and 'Maculata' produce green leaves with white margins; 'Tricolor' has deep-green, lightgreen, and cream variegated foliage; 'Quadricolor' has deepgreen, light-green, cream, and yellow variegated foliage; and 'Lemon Wave' has bright-yellow splotches on variegated green-and-white leaves (Dirr, 2004; van Gelderen and van 
Gelderen, 2004). We found no differences among 'Lemon Wave', 'Maculata', and two nonvariegated cultivars, Mariesii Perfecta and Seafoam. 'Lemon Wave' is a sport derived from 'Seafoam' (van Gelderen and van Gelderen, 2004), so it is not surprising that our markers did not reveal differences between these two cultivars. We propose that 'Maculata' may also be a sport of one of the members of the clade. The presence of 'Mariesii Perfecta' in this clade is somewhat more difficult to explain. Although 'Seafoam' is an old cultivar introduced from Japan, 'Mariesii Perfecta' was selected from open-pollinated seedlings of 'Mariesii' (Haworth-Booth, 1984). However, 'Mariesii' was also an early introduction from Japan and shows considerable genetic similarity to 'Seafoam'. Although the lack of discernible differences between 'Mariesii Perfecta' and 'Seafoam' may be the result of mislabeling of plants, it is also possible that it is merely a reflection of the close genetic similarity between 'Seafoam' and at least one parent of 'Mariesii Perfecta'. Additional or different markers may have revealed differences between 'Seafoam' and 'Mariesii Perfecta'.

The other three variegated cultivars exhibited close genetic similarity. 'Tricolor' has been proposed to be a branch sport of 'Mariesii'; however, our results do not indicate this to be correct. Also, despite its name, 'Mariesii Variegata' does not appear to be a sport of 'Mariesii' or of 'Mariesii Perfecta', 'Whitewave' (synonym, 'Mariesii Grandiflora'), or 'Lilicina' (synonym, 'Mariesii Lilicina'), all of which are open-pollinated selections of 'Mariesii' (Haworth-Booth, 1984).

One of the most interesting findings of this study was that, with a few exceptions that will be discussed later, mophead and lacecap cultivars clustered separately in the PCoA (Fig. 1). We interpret this finding to mean that neither the lacecap cultivars that were among the earliest introductions from Japan to Europe (e.g., 'Mariesii' and 'Veitchii') nor their offspring were used extensively by European breeders in the development of mophead cultivars. The H. macrophylla ssp. macrophylla gene pool currently available in the United States may be narrow, but it also appears that it may not have been fully exploited. Many of the lacecap cultivars may have valuable traits, such as disease resistance, lacking in the mophead cultivars. A prime example of this is the powdery mildew resistance that has been reported for 'Veitchii' (Dirr, 2004). Although lacecap inflorescence form appears to be dominant, this trait seems to be conditioned by only one or two genes. Some lacecap cultivars, including the cultivar Veitchii, appear to be heterozygous for inflorescence form (S.M. Reed, unpublished data), allowing mophead inflorescence form to be recovered in the $F_{1}$ generation. As additional lacecap cultivars are imported to the United States from Europe and Asia, it is important to evaluate them for useful genes that could be exploited by breeding programs.

An exception to the apparent previous lack of use of lacecap cultivars in the breeding of mophead cultivars involves white flower color. Three white-flower mophead cultivars fell outside the main mophead cluster. Although the parentage of 'Soeur Therese' and 'Princess Juliana' is unknown, 'Madame Emile Mouillere' is a hybrid of 'Rosea' and 'Mariesii Grandiflora' (Haworth-Booth, 1984). 'Mariesii Grandiflora' is a whiteflower lacecap cultivar that originated as an open-pollinated seedling of 'Mariesii'. We propose that white flower color was incorporated into the mophead cultivars from 'Mariesii Grandiflora' or other white-flower lacecap cultivars.
The 10 lacecap cultivars that fell within the mophead cluster are all members of the Teller Series, a group of lacecap cultivars released by the Federal Research Institute for Horticulture in Switzerland from 1952 to 1987. Although some of the mophead cultivars in the cluster are of unknown origin, most of the others were introduced before the Teller Series cultivars. The Teller Series cultivars were bred by crossing a mophead with a lacecap cultivar (Bell and Bell, 1997; Dirr, 2004). Although exact parentage for these cultivars has not been published, both 'Todi' and 'Enziandom' have been mentioned as parents (Bell and Bell, 1997; van Gelderen and van Gelderen, 2004). The only Teller Series cultivar we examined that did not fall within the mophead cluster was 'Libelle', a white-flower cultivar.

Almost all the H. macrophylla ssp. macrophylla cultivars included in this study were either early imports from Japan to Europe, bred in Europe before World War II, or of unknown origin. However, recently introduced $H$. macrophylla ssp. macrophylla cultivars from Europe ('Hobella' and 'Shamrock') and Japan ('Hanabi', 'Izo-no-Hana', 'Jogosaki', 'Fuji Waterfall', and 'Frau Fujijo') were also examined. 'Hobella' produces lacecap inflorescences and originated from controlled pollinations of two unnamed seedlings (U.S. Patent Office, 1996). It showed no close genetic similarity to any other cultivar in this study. 'Shamrock' produces double-flower lacecap inflorescences and originated from an open-pollination of an unnamed seedling (U.S. Patent Office, 2006). 'Hanabi', 'Izo-no-Hana', and 'Jogosaki' are also double-flower lacecap cultivars and exhibited genetic similarities to each other and to 'Shamrock'. 'Izo-no-Hana' and 'Jogasaki' were discovered on the east coast of Izu, but the greatest genetic similarity within this group was found between 'Izo-no-Hana' and 'Hanabi', an old cultivar whose origin is unknown (Yamamoto, 2000). 'Fuji Waterfall' is a double-flower cultivar that was discovered near Mt. Fuji (Yamamoto, 2000). It did not lie in the cluster with the other four double-flower $H$. macrophylla ssp. macrophylla cultivars. No close genetic relationships between the new double-flower introductions and any of the early European cultivars were observed. The final new introduction from Japan that was included in this study, 'Frau Fujiyo', is a mophead cultivar that was bred from controlled pollinations (U.S. Patent Office, 1998). Although 'Frau Fujiyo' showed no close genetic similarity to any other cultivar in our study, it did fall within the mophead cluster in the PCoA. It appears that some of the new H. macrophylla ssp. macrophylla introductions from Europe and Japan may represent a widening of the genetic base available in this country for breeding purposes.

This study included six sets of cultivars that have been proposed to represent synonyms. 'Geoffrey Chadbund' is listed by Bertrand (2001) and Dirr (2004) as a synonym for 'Mowe', but Mallet (1994) and van Gelderen and van Gelderen (2004) indicate that they are different cultivars. According to the latter two references, 'Mowe' was released by the Federal Research Institute for Horticulture in Switzerland in 1964, whereas Russell introduced 'Geoffrey Chadbund' before 1956. Photographs and descriptions of the two cultivars are very similar (Lawson-Hall and Rothera, 1995; van Gelderen and van Gelderen, 2004). With the exception of missing loci, the 'Geoffrey Chadbund' specimen that we analyzed was genetically identical to 'Mowe'. It is possible that, because of their very similar phenotypes, these two cultivars have become 
confused in the trade. 'Preziosa' and 'Pink Beauty' produced $100 \%$ identical alleles, suggesting that these plants have been correctly assumed to be the same cultivar. All references consulted agreed that 'Preziosa' and 'Pink Beauty' are synonyms, although this was based solely on the distinct phenotype of these plants.

'Harlequin' and 'Monrey' are listed as synonyms in some publications (Bertrand, 2001; Dirr, 2004). 'Harlequin', which also is listed by the synonym 'Sensation 75', was bred in Germany and introduced in 1957 (van Gelderen and van Gelderen, 2004). 'Monrey' ('Buttons 'n Bows') was discovered as a branch sport in 1992 at Monrovia Nursery (U.S. Patent Office, 2000). Results of our study indicate that 'Harlequin' and 'Monrey', with a minimum divergence estimate of $1 \%$, differ at several loci and are different cultivars.

Two sets of possible synonyms showed considerable genetic similarity, but did not produce $100 \%$ identical alleles. 'Ayesha' and 'Uzu' differed at only one allele, where 'Ayesha' was homozygous and 'Uzu' was heterozygous, and had a minimum genetic divergence estimate of $0.6 \%$. Analysis of these two specimens using different markers might reveal greater genetic differences. However, it is possible that the specimens of 'Ayesha' and 'Uzu' we used originated from the same cultivar. If they were maintained separately, accumulations of mutations over time could have occurred, resulting in minor differences in SSR alleles. The same is true for 'Rosalba' and 'Benigaku', which also only differed at one locus by one allele and had a minimum genetic distance of $0.7 \%$. Although there is disagreement among authorities on whether 'Rosalba' and 'Benigaku' are the same cultivar, our data suggest that they are the same.

The minimum genetic distance estimates for the remaining possible pair of synonyms, 'Mandschurica' and 'Nigra', was $4.1 \%$. Even though the genetic differences were not great, we believe them to be separate cultivars. Mallet et al. (1992) list 'Nigra' as a Chinese horticultural variety imported to Europe by Wilson and given the name $H$. macrophylla var. mandschurica. The U.S. National Arboretum website states that 'Nigra' was purchased during a 1985 expedition to Japan (U.S. National Arboretum, 2006). According to this source, 'Nigra' had been in cultivation in England since the late 1800s. It was released to the American nursery trade by the U.S. National Arboretum in 1993. Based on the results of our study, it appears that there is more than one black-stem cultivar available in the trade; however, the 'Nigra' and 'Mandschurica' specimens that we examined did exhibit considerable genetic similarities.

We were surprised to find that several sets of cultivars that are not listed as synonyms had 100\% identical genotypes. Similarities within the remontant and variegated cultivars have already been discussed, but we also found no differences between 'Blaumeise' and 'Eisvogel' or between 'Fasan' and 'Zaunkoenig'. These four cultivars are all part of the Teller Series of cultivars and may have similar parentage. Additional markers might discern differences between each pair of cultivars. However, most of the members of the Teller Series have similar phenotypes, producing large lacecap inflorescences with large, deeply colored imperfect flowers and deep-green foliage. Some of them could be easily confused, particularly if they were grown in the absence of aluminum, where all would produce pink flowers. In addition, Teller Series cultivars have been offered for sale in the United States under the incorrect names 'Teller Blue', 'Teller Red', 'Teller Pink', and 'Teller
Purple'. Therefore, it is possible that one or more of the specimens we examined were mislabeled.

All conclusions made in this study are based on the assumptions that the plant material we analyzed was correctly labeled. Considering how long some of the H. macrophylla cultivars have been in existence and the similarities in phenotype among many cultivars, it would be surprising if there were no misidentified plants in our study. Although some of the relationships that we identified between specific sets of cultivars may not be correct as a result of misidentified plant materials, we feel that the overall trends we identified are valid. Our greatest concern is with the oldest cultivars, such as Rosea, Sir Joseph Banks, and Otaska. We are currently seeking other sources of these cultivars, but unfortunately these are not commonly available in the United States.

In summary, SSR markers were used successfully to analyze genetic diversity in $H$. macrophylla. In addition to providing information relative to the taxonomic classification of $H$. macrophylla, this study identified genetic relationships between cultivars and potentially unexploited sources of germplasm for improvement of this popular shrub. It is hoped that this information will provide direction to breeders attempting to develop new, improved forms of H. macrophylla.

\section{Literature Cited}

Bailey, D.A. 1989. Hydrangea production. Timber Press, Portland, Ore.

Bell, A. and T. Bell. 1997. Special names for special plants. Amer. Nurseryman 186:86-91.

Bertrand, H. 2000. Management and knowledge of the Hydrangea collection of Angers: Morphological characters and data analysis. Acta Hort. 508:173-178.

Bertrand, H. 2001. Preliminary Hydrangea checklist. Institut National d'Horticulture, Angers, France.

Dirr, M.A. 2004. Hydrangeas for American gardens. Timber Press, Portland, Ore.

Goudet, J. 1995. FSTAT (version 1.2): A computer program to calculate F-statistics. J. Hered. 86:485-486.

Hara, H. 1955. Critical notes on some type specimens of east Asiatic plants in foreign herbaria. J. Jpn. Bot. 30:271-278.

Haworth-Booth, M. 1984. The hydrangeas. 5th ed. Constable, London.

Jin, L. and R. Chakraborty. 1994. Estimation of genetic distance and coefficient of gene diversity from single-probe multilocus DNA fingerprinting data. Mol. Biol. Evol. 11:120-127.

Jones, K.D., S.M. Reed, and T.A. Rinehart. 2007. Analysis of ploidy level and its effects on guard cell length, pollen diameter and fertility in Hydrangea. HortScience 42:483-488.

Langella, O. 2002. Populations: A free population genetics software. 28 Feb. 2006. <www.pge.cnrs-gif.fr/bioinfo/populations/index. php?lang $=$ en $\&$ doc $=$ populations $>$.

Lawson-Hall, T. and B. Rothera. 1995. Hydrangeas: A gardeners guide. Timber Press, Portland, Ore.

Levin, D.A. 2002. The role of chromosomal change in plant evolution. Oxford University Press, New York.

Lindstrom, J.T., M.C. Pelto, and M.A. Dirr. 2003. Molecular assessment of remontant (reblooming) Hydrangea macrophylla cultivars. J. Environ. Hort. 21:57-60.

Mallet, C. 1994. Hydrangeas: Species and cultivars. Vol. 2. Centre d'Art Floral, Varengeville, France.

Mallet, C., R. Mallet, and H. van Trier. 1992. Hydrangeas: Species and cultivars. Vol. 1. Centre d'Art Floral, Varengeville, France.

McClintock, E. 1957. A monograph of the genus Hydrangea. Proc. Calif. Acad. Sci. 29:147-256. 
Nei, M. 1972. Genetic distance between populations. Amer. Naturalist 106:283-292.

Nei, M. 1973. Analysis of gene diversity in subdivided populations. Proc. Natl. Acad. Sci. USA 70:3321-3323.

Page, R.D. 1996. TreeView: An application to display phylogenetic trees on personal computers. Comput. Appl. Biosci. 12:357-358.

Reed, S.M. 2000. Development of an in ovolo embryo culture procedure for Hydrangea. J. Environ. Hort. 18:34-39.

Reed, S.M. 2005. Pollination biology of Hydrangea macrophylla. HortScience 40:335-338.

Reed, S.M. and T.A. Rinehart. 2006. Hydrangea macrophylla and serrata: Should we lump 'em or split 'em? Proc. Southern Nursery Assoc. Res. Conf. 51:573-576.

Rinehart, T.A., B.E. Scheffler, and S.M. Reed. 2006. Genetic diversity estimates for the genus Hydrangea and development of a molecular key based on SSR. J. Amer. Soc. Hort. Sci. 131:787-797.

Rohlf, F.J. 1992. NTSYS-pc numerical taxonomy and multivariate analysis system, version 1.70. Exeter Software, Setauket, N.Y.

Saitou, N. and M. Nei. 1987. The neighbor-joining method: A new method for reconstructing phylogenetic trees. Mol. Biol. Evol. 4:406-425.

Stephens, J.C., D.A. Gilbert, N. Yuhki, and S.J. O'Brien. 1992. Estimation of heterozygosity for single-probe multilocus DNA fingerprints. Mol. Biol. Evol. 9:729-743.

U.S. National Arboretum. 2003. Hydrangea macrophylla 'Nigra'. 14 June 2006. <www.usna.usda.gov/Newintro/hydrang1.html>.

U.S. Patent Office. 1996. Hydrangea plant named Hobella. 17 July 2006. <http://patft.uspto.gov/netacgi/nph-Parser?Sect1=PTO2\& Sect $2=$ HITOFF \& $p=1 \& u=\% 2$ Fnetahtm $1 \% 2$ FPTO $\% 2$ Fsearch- bool.html\& $\mathrm{r}=1 \& \mathrm{f}=\mathrm{G} \& 1=50 \& \mathrm{col}=\mathrm{AND} \& \mathrm{~d}=\mathrm{PTXT} \& \mathrm{~s} 1=$ Hobella\& OS=Hobella\&RS=Hobella $>$

U.S. Patent Office. 1998. Hydrangea plant named Frau Fujiyo. 17 July 2006. <http://patft.uspto.gov/netacgi/nph-Parser?Sect1=PTO2\& Sect $2=$ HITOFF $\& p=1 \& u=\% 2$ Fnetahtm $1 \% 2$ FPTO $\% 2$ Fsearch-

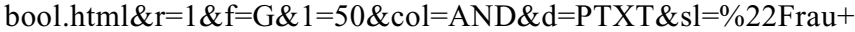
Fujiyo $\% 22 \& O S=>$

U.S. Patent Office. 2000. Hydrangea plant named Monrey. 17 July 2006. $<$ http://patft.uspto.gov/netacgi/nph-Parser?Sect1=PTO2\& Sect $2=$ HITOFF $\& p=1 \& u=\% 2$ Fnetahtm $1 \% 2$ FPTO $\% 2$ Fsearchbool.html\&r=2\&f=G\&1=50\&col=AND\&d=PTXT\&s1=monrey\&OS= monrey\&RS $=$ monrey $>$

U.S. Patent Office. 2006. Hydrangea plant named Shamrock. 17 July 2006. <http://patft.uspto.gov/netacgi/nph-Parser?Sect1=PTO2\& Sect $2=$ HITOFF $\& p=1 \& u=\% 2$ Fnetahtml $\% 2$ FPTO $\% 2$ Fsearch-bool. $\mathrm{htm} l \& \mathrm{r}=2 \& \mathrm{f}=\mathrm{G} \& 1=50 \& \mathrm{co} 1=\mathrm{AND} \& \mathrm{~d}=\mathrm{PTXT} \& \mathrm{~s} 1=$ shamrock $\& \mathrm{~s} 2=$ PP\$.PN.\&OS=shamrock+AND+PN/PP\&RS=shamrock + AND + PN/ $\mathrm{PP}>$

van Gelderen, C.J. and D.M. van Gelderen. 2004. Encyclopedia of hydrangeas. Timber Press, Portland, Ore.

Wilson, E.H. 1923. The hortensias: H. macrophylla Seringe and H. serrata (Thunberg). Seringe. J. Arnold Arboretum 4:233-246.

Yamamoto, T. 2000. The Japanese hydrangeas color guide book. Association Shamrock, Varengeville-sur-Mer, France. [English translation].

Zonneveld, B.J.M. 2004. Genome size in Hydrangea, p. 245-251. In: C.J. van Gelderen and D.M. van Gelderen (eds.). Encyclopedia of hydrangeas. Timber Press, Portland, Ore. 